\title{
Espiritualidade no cuidado ao paciente oncológico em processo de morte: percepção dos profissionais de enfermagem
}

\author{
Spirituality in the care of cancer patients in the process of dying: nursing professionals' \\ perception
}

\section{Espiritualidad en cuidado de pacientes oncologico en proceso de muerte: percepción de profesionales de enfermería}

Thayenne Barrozo Mota Monteiro ${ }^{1 *}$, Manuela Gomes Campos Borel2 ${ }^{2}$, Isabel Cristina de Oliveira Arrieira ${ }^{3}$, Fábio da Costa Carbogim¹, Maira Buss Thofehrn ${ }^{4}$, Thaís Vasconselos Amorim¹.

\section{RESUMO}

Objetivo: Identificar a percepção dos profissionais de enfermagem quanto à espiritualidade no âmbito do cuidado ao paciente oncológico em processo de morte. Métodos: Revisão integrativa nas bases de dados da BDEnf, Lilacs, Medline, Pubmed a partir da Biblioteca Virtual de Saúde, baseada nos descritores espiritualidade, enfermagem, oncologia, assistência terminal, terminalidade, perfazendo uma amostra final de 07 artigos científicos selecionados. As buscas dos dados foram realizadas de janeiro a março de 2019. Resultados: A espiritualidade para a equipe de enfermagem elencou-se em reconhecer que a dimensão espiritual no cuidado do paciente oncológico que vivencia o processo de morte, proporciona mais sentido ao trabalho, favorece a relação intrínseca e extrínseca, além de fortalecer os vínculos entre profissionais, pacientes, familiares e acompanhantes. Considerações finais: Necessário mais investimentos em pesquisas que comprovem os impactos que o cuidado espiritual pode acarretar ao paciente e que há uma precariedade de profissionais capacitados para desenvolverem esse cuidado.

Palavras-chave: Assistência terminal, Oncologia, Espiritualidade, Enfermagem.

\begin{abstract}
Objective: Identify the perception of nursing professionals regarding spirituality in the context of care for cancer patients in the process of death. Methods: Integrative review in the databases of BDEnf, Lilacs, Medline, Pubmed from the Virtual Health Library, based on the descriptors spirituality, nursing, oncology, terminal assistance, terminality, making a final sample of 07 selected scientific articles. Data searches were carried out from January to March of 2019. Results: Spirituality for the nursing team was recognized in recognizing that the spiritual dimension in the care of cancer patients who experience the death process, provides more meaning to work, favors the intrinsic and extrinsic relationship, in addition to strengthening the bonds between professionals, patients, family members and companions. Final considerations: More investments in research are needed to prove the impacts that spiritual care can have on the patient and that there is a precariousness of trained professionals to develop this care.
\end{abstract}

Key words: Terminal care, Medical oncology, Spirituality, Nursing.

\section{RESUMEN}

Objetivo: Identificar la percepción de los profesionales de enfermería sobre la espiritualidad en el contexto de la atención al paciente oncológico en proceso de muerte. Métodos: Revisión integrativa en las bases de datos de BDEnf, Lilacs, Medline, Pubmed de la Biblioteca Virtual en Salud, con base en los descriptores espiritualidad, enfermería, oncología, asistencia terminal, terminalidad, realizando una muestra final de 07 artículos científicos seleccionados. Las búsquedas de datos se realizaron de enero a marzo de 2019.

\footnotetext{
${ }^{1}$ Universidade Federal de Juiz de Fora (UFJF), Juiz de Fora - MG. *E-mail: thayennemonteiro@yahoo.com.br

${ }^{2}$ Universidade Federal do Rio de Janeiro (UFRJ), Rio de Janeiro - RJ.

${ }^{3}$ Universidade Católica de Pelotas (UCPel), Pelotas - RS.

${ }^{4}$ Universidade Federal de Pelotas (UFPel), Pelotas - RS.
} 
Resultados: Se destacó la espiritualidad para el equipo de enfermería al reconocer que la dimensión espiritual en el cuidado del paciente oncológico que vive el proceso de muerte, da más sentido al trabajo, favorece lo intrínseco y extrínseco. relación, además de fortalecer los lazos entre profesionales, pacientes, familiares y acompañantes. Consideraciones finales: Se necesitan más inversiones en investigación para demostrar los impactos que puede tener el cuidado espiritual en el paciente y que existe una precariedad de profesionales capacitados para desarrollar este cuidado.

Palabras clave: Cuidado terminal, Oncología médica, Espiritualidad, Enfermería.

\section{INTRODUÇÃO}

Ao considerar que os seres humanos são constituídos por dimensões físicas, emocionais, sociais e espirituais, o cuidado à saúde deve ser desenvolvido numa perspectiva holística (BUBLOZ BK, et al., 2019). Tal valorização das dimensões dos seres humanos torna-se ainda mais essencial quando se trata de pacientes oncológicos. Porque a oncologia recebe cada vez mais destaque, em virtude de ser considerada um dos problemas mais complexos de saúde pública que apresenta expressiva relevância epidemiológica, social e econômica no sistema de saúde (BUBLOZ BK, et al., 2019).

Diante do exposto, o cuidado ao paciente oncológico deve valorizar as suas diversas dimensões, e quando se trata daqueles em processo de morte, o envolvimento da espiritualidade auxilia na aceitação da sua finitude, em compreendê-la como parte do percurso natural da vida. Ainda, cabe dizer que a consciência da morte e o seu respeito otimiza no indivíduo, a vontade de viver plenamente e aceitar com mais facilidade o seu estado de saúde (KUBLER-ROSS E, 2017). Embora, sem desconsiderar os sentimentos de medo frente ao desconhecido que o processo da morte pode desencadear, cabe ao profissional de enfermagem amparar o paciente nesse processo por meio da promoção de apoio, conforto, segurança (KUBLER-ROSS E, 2017).

Vale ressaltar que a enfermagem vivencia um paradigma político-social que defende a vertente de que a ocorrência da morte deve ser evitada a todo custo, sendo esse, um dos motivos pelo qual os profissionais manifestam sofrimentos diante do processo de morte de seus pacientes (BASTOS RA, et al., 2018).

Tais manifestações podem ser justificadas pelo fato de que esses profissionais que cuidam da dor alheia, não receberam o acolhimento adequado para os seus próprios sofrimentos e limitações diante do processo da morte, o que pode desencadear o adoecimento do trabalhador. E, para melhor assistir àquele que vivencia o processo de morte, cabe aos profissionais aprenderem a considerar o momento da morte como um fenômeno complexo que exige um esforço cognitivo e emocional. Ficando evidente a necessidade de um preparo adequado, porém, a abordagem sobre a morte ainda é incipiente no processo de formação, em especial, na graduação em enfermagem, o que determina a necessidade de um maior investimento das instituições de ensino para sanar essa lacuna (LIMA R, et al., 2017).

Por conseguinte, diante da demanda requerida, pelo âmbito oncológico, o cuidado envolve além da dimensão física, pessoal, interrelações familiares, sociais e espirituais, requer que os profissionais estejam preparados para enfrentarem situações frente ao processo de morte de seus pacientes (BUBLOZ BK, et al., 2019). Assim, torna-se substancial o equilíbrio emocional em prol de uma assistência segura e de qualidade aos envolvidos. Nessa perspectiva, o cuidado na contemporaneidade tem envolvido a valorização da dimensão espiritual à finitude de vida que compreende questões particulares influenciadas pelas culturas e vivências, embora ainda existam vertentes que confundem espiritualidade e religiosidade (CATRÉ MNC, et al., 2016; MIQUELETTO M, et al., 2017).

Sendo assim, a pesquisa apresenta como lacuna a incipiência do cuidado espiritual ao paciente oncológico em processo de morte desempenhado pelos profissionais de enfermagem. Tal constatação justifica o interesse em elaborar uma Revisão Integrativa (RI) que envolva a equipe de enfermagem diante do paciente oncológico em processo de morte, com a finalidade de promover reflexões e identificar possíveis conceitos inovadores sobre o impacto da espiritualidade para a área. Isto posto, o estudo teve como objetivo identificar a percepção dos profissionais de enfermagem quanto à espiritualidade no âmbito do cuidado ao paciente oncológico em processo de morte. 


\section{MÉTODOS}

Trata-se de um estudo com abordagem qualitativa desenvolvido por meio da estratégia de revisão integrativa em seis etapas: 1) definição da pergunta de pesquisa; 2) definição dos critérios de inclusão dos estudos científicos e escolha da amostra; 3) exibição dos estudos pertinentes em tabelas; 4) realização da análise crítica; 5) interpretação dos resultados; e por fim, 6) atribuição das evidências encontradas de maneira clara (RUIVO BARA, et al., 2020; QUIJADA PDS, et al., 2018).

Para a primeira etapa, definição da pergunta de pesquisa, foi realizado um aprofundamento teórico a respeito da espiritualidade da enfermagem (CHIANG YC, et al., 2015) oncológica no processo de morte em literaturas existentes, o que permitiu obter uma compreensão e definir como pergunta de pesquisa: "qual a produção do conhecimento em enfermagem que versa sobre a percepção de espiritualidade dos profissionais de enfermagem diante do paciente oncológico em processo de morte?".

$\mathrm{Na}$ segunda etapa, definição dos critérios de inclusão dos estudos científicos e escolha da amostra, a coleta de dados ocorreu entre janeiro e março de 2019, foram selecionados estudos considerados primários, baseados na pergunta de pesquisa e critérios de inclusão pré-definidos. Foram utilizados critérios de inclusão baseados em estudos com abordagem quantitativa ou qualitativa, restringidos ao âmbito da enfermagem.

Portanto, os estudos selecionados seguiram as seguintes atribuições elencadas: ser artigo científico, apresentar publicação nos idiomas, português, inglês ou espanhol, o período de publicação entre 2015 e 2019, o texto completo estar disponível na íntegra nas bases de dados da Biblioteca Virtual de Saúde (BVS), adequar-se à temática de espiritualidade e processo de morte do paciente oncológico; e ter como públicoalvo, a equipe de enfermagem. Além disso, é importante destacar, que "AND" foi o operador booleano de escolha entre os descritores, assistência terminal, terminalidade, oncologia, espiritualidade e enfermagem.

Vale salientar ainda, que os estudos publicados que não acatavam os devidos critérios, foram excluídos; àqueles em que não abordavam a temática de escolha, não apresentavam a descrição metodológica de maneira clara, sem resumo disponível nas bases de dados selecionadas, relacionados a outros profissionais da área da saúde e os que se apresentaram repetidos.

A seleção dos descritores foi alicerçada nas expressões do Descritores em Ciências da Saúde (DeCS) que é um dicionário elaborado pelo Centro-Latino-Americano e do Caribe de Informação em Ciências da Saúde, com o intuito de padronizar a linguagem na descrição de publicações científicas, que favorece e facilita organizar assuntos de mesma temática na literatura. A estratégia de busca compreendeu os recursos informacionais de bases de dados eletrônicas (Quadro 1).

Quadro 1 - Filtros para refinar a busca dos dados.

\begin{tabular}{|c|c|}
\hline 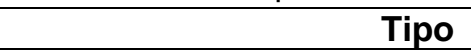 & $\begin{array}{l}\text { Filtros da pesquisa } \\
\end{array}$ \\
\hline Descritor & $\begin{array}{l}\text { Espiritualidade } \\
\text { Enfermagem } \\
\text { Oncologia } \\
\text { Terminalidade } \\
\text { Assistência Terminal }\end{array}$ \\
\hline Base de dados & $\begin{array}{l}\text { Bdenf } \\
\text { Medline } \\
\text { Pubmed } \\
\text { Lilacs }\end{array}$ \\
\hline Assunto principal & $\begin{array}{l}\text { Espiritualidade } \\
\text { Equipe de Enfermagem } \\
\text { Paciente oncológico em processo de morte }\end{array}$ \\
\hline Ano de publicação & $2015-2019$ \\
\hline Texto & Disponível e completo na íntegra \\
\hline Idiomas & Português - Inglês - Espanhol \\
\hline Tipo de documento & Artigo científico \\
\hline
\end{tabular}

Fonte: Monteiro TBM, et al., 2021. 
Foram avaliados os títulos e resumos, e aqueles em que tanto o título quanto o resumo não foram suficientes para a realização da seleção inicial, tornou-se necessária à sua leitura na íntegra.

Dos conteúdos obtidos e a partir das buscas nas distintas bases de dados bibliográficos, considerou-se necessário o emprego de filtros para a seleção de estudos selecionados.

Ressalva-se que o atual estudo não envolveu seres humanos, portanto, não foi necessária avaliação prévia da pesquisa pelo Comitê de Ética em Pesquisa com Seres Humanos, mas foram seguidos rigorosamente os cuidados éticos, principalmente o que tange a plágio e direitos autorais, na busca, análise, discussão e apresentação dos resultados.

\section{RESULTADOS E DISCUSSÃO}

A partir das buscas nas distintas bases de dados bibliográficos, considerou-se necessário o emprego de filtros para a seleção de estudos identificados em cada base. A primeira busca selecionou 657.588 publicações e posteriormente, com a utilização dos critérios de inclusão, restaram 6.775 artigos. A seguir, o fluxograma empregado para organizar os artigos respeitando as fases desta revisão integrativa, ilustrados na (Figura 1).

Figura 1 - Etapas referentes à seleção dos artigos relevantes para a revisão integrativa.

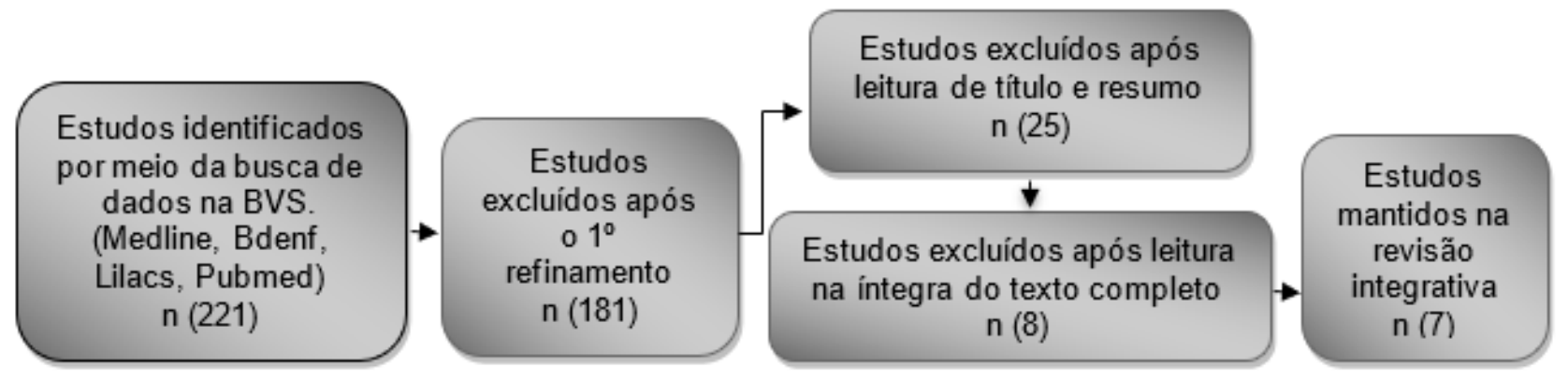

Fonte: Monteiro TBM, et al., 2021.

Dessa forma, após a investigação inicial que tornou viável a identificação de 221 estudos, seguiu-se para o refinamento de busca e aplicação dos critérios de inclusão e exclusão, leitura de título e resumo, e de texto na íntegra, totalizando para a amostra desta revisão, 7 artigos.

Foi excluído um artigo que se encontrava repetido entre os descritores "Espiritualidade AND Enfermagem AND Assistência Terminal", exibido tanto na base da Lilacs quanto na Medline. Outro artigo, também duplicado, porém entre "Espiritualidade AND Enfermagem AND Oncologia", na Bdenf e na Lilacs. Por fim, na associação de "Espiritualidade AND Enfermagem AND Terminalidade", um artigo apresentava-se em português e inglês. Dessa forma, duplicado para a análise final.

Portanto, os 7 artigos desta revisão, todos publicados em periódicos indexados sendo $3(43 \%)$ em periódicos internacionais e $4(57 \%)$ em periódicos nacionais, os artigos são provenientes da China 1(14\%), EUA 1(14\%), Austrália 1 (14\%) e, do Brasil 4 (57\%). Os artigos são dos anos de 2015, 2016 e 2017 sendo 2 artigos em cada ano e, 01 em 2018; apesar do universo de artigos incluir de 2019 não foram selecionados, deste ano, na amostra. Em relação a categoria profissional dos autores $6(86 \%)$ foram escritos por autores enfermeiros e, $1(14 \%)$ em parceria com profissionais atuantes na oncologia, como médicos. No que tange ao desenho dos estudos, 2 (29\%) são pesquisas com abordagem quantitativos e 5 (71\%) com abordagem qualitativa, neste último estão inclusas todas as pesquisas de origem do Brasil.

E mediante as associações entre os descritores combinados pelo operador booleano "AND", além de outros critérios de inclusão e exclusão, o total de artigos na amostra também se deu por meio da combinação a seguir.

Espiritualidade AND Enfermagem AND Terminalidade, detectou 42 artigos, Espiritualidade AND Enfermagem AND Oncologia, 22, e entre Espiritualidade AND Enfermagem AND Assistência Terminal, 157 
artigos científicos. Essas, definidas como as combinações mais específicas para o alcance do objetivo da pesquisa, no qual se pauta em envolver a espiritualidade e a enfermagem na assistência ao paciente oncológico que vivencia o seu processo de morte.

A partir dos 7 estudos incluídos foi elaborado um quadro contendo características, objetivos e conclusões dos artigos selecionados, apresentado a seguir no Quadro 2.

Quadro 2 - Resultado da análise dos dados coletados dos estudos selecionados para a revisão integrativa

\begin{tabular}{|c|c|c|c|c|c|}
\hline Ano & Autores & Cidade & Periódico & Objetivos & Conclusões \\
\hline 2015 & $\begin{array}{l}\text { CHIANG YC, } \\
\text { et al. }\end{array}$ & $\begin{array}{l}\text { Taiwan, } \\
\text { China. }\end{array}$ & $\begin{array}{l}\text { Nursing } \\
\text { Outlook }\end{array}$ & $\begin{array}{l}\text { Investigar o impacto da } \\
\text { saúde espiritual dos } \\
\text { enfermeiros referente ao } \\
\text { cuidado espiritual praticado } \\
\text { pelos mesmos. }\end{array}$ & $\begin{array}{l}\text { A saúde espiritual desses } \\
\text { profissionais deve ser vista como } \\
\text { um impactante valor e sistema de } \\
\text { crenças que pode influenciar em } \\
\text { seu desempenho profissional. }\end{array}$ \\
\hline 2015 & $\begin{array}{l}\text { RODIN D, et } \\
\text { al. }\end{array}$ & $\begin{array}{l}\text { Boston, } \\
\text { Estados } \\
\text { Unidos. }\end{array}$ & $\begin{array}{c}\text { Support } \\
\text { Care Cancer }\end{array}$ & $\begin{array}{l}\text { Identificar como } \\
\text { enfermeiros e médicos na } \\
\text { oncologia consideram o } \\
\text { seu papel no cuidado } \\
\text { espiritual (CE), quais os } \\
\text { fatores interferem na } \\
\text { percepção e como a crença } \\
\text { afeta o fornecimento do CE. }\end{array}$ & $\begin{array}{l}\text { Os enfermeiros foram mais } \\
\text { propícios a fornecerem os CE } \\
\text { comparados aos médicos. O papel } \\
\text { dos médicos em promover esses } \\
\text { cuidados está atrelado às questões } \\
\text { religiosas e espirituais. } \\
\text { treinamento em CE pode favorecer } \\
\text { no aumento de seu fornecimento. }\end{array}$ \\
\hline 2016 & $\begin{array}{l}\text { EVANGELIS } \\
\text { TA CB, et al. }\end{array}$ & $\begin{array}{l}\text { João } \\
\text { Pessoa, } \\
\text { Brasil. }\end{array}$ & $\begin{array}{c}\text { Esc Anna } \\
\text { Nery }\end{array}$ & $\begin{array}{l}\text { No contexto dos } \\
\text { enfermeiros, o objetivo } \\
\text { envolveu a compreensão } \\
\text { da espiritualidade no } \\
\text { assistir do paciente em } \\
\text { cuidado paliativo. }\end{array}$ & $\begin{array}{l}\text { O estudo concluiu que ainda existe } \\
\text { uma falta de preparação dos } \\
\text { enfermeiros em se tratar de } \\
\text { questões espirituais nos cuidados } \\
\text { paliativos, embora reconheçam a } \\
\text { relevância da dimensão espiritual } \\
\text { na execução dos seus cuidados. }\end{array}$ \\
\hline 2016 & $\begin{array}{l}\text { SILVA BS, et } \\
\text { al. }\end{array}$ & $\begin{array}{l}\text { Cidade do } \\
\text { sudeste } \\
\text { brasileiro. }\end{array}$ & $\begin{array}{l}\text { Cogitare } \\
\text { Enferm. }\end{array}$ & $\begin{array}{l}\text { Apurar a percepção da } \\
\text { espiritualidade pela } \\
\text { enfermagem referente aos } \\
\text { cuidados no final da vida. }\end{array}$ & $\begin{array}{l}\text { O cuidado espiritual na finitude da } \\
\text { vida é incipiente, embora a } \\
\text { enfermagem demonstre boa } \\
\text { aceitação da morte. É fundamental } \\
\text { envolver a temática na graduação e } \\
\text { curso técnico de enfermagem, e } \\
\text { investir em estudos para capacitar e } \\
\text { sensibilizar o profissional de } \\
\text { enfermagem. }\end{array}$ \\
\hline 2017 & $\begin{array}{l}\text { SIQUEIRA } \\
\mathrm{HCH} \text {, et al. }\end{array}$ & $\begin{array}{l}\text { Pelotas, } \\
\text { Brasil. }\end{array}$ & $\begin{array}{l}\text { Rev enferm } \\
\text { UFPE } \\
\text { online. }\end{array}$ & $\begin{array}{l}\text { Investigar o impacto da } \\
\text { espiritualidade no processo } \\
\text { saúde-doença na oncologia } \\
\text { sob a percepção do } \\
\text { enfermeiro. }\end{array}$ & $\begin{array}{l}\text { É fundamental investigar, analisare } \\
\text { colocar em prática o cuidado } \\
\text { espiritual no processo saúde- } \\
\text { doença a partir da sua inserção na } \\
\text { graduação de enfermagem além de } \\
\text { disseminar na assistência em } \\
\text { saúde em geral. }\end{array}$ \\
\hline 2017 & $\begin{array}{l}\text { RONALDSO } \\
\text { N S, et al. }\end{array}$ & $\begin{array}{c}\text { Sydney } \\
\text { Austrália. }\end{array}$ & $\begin{array}{l}\text { International } \\
\text { Journal of } \\
\text { Palliative } \\
\text { Nursing. }\end{array}$ & $\begin{array}{l}\text { Investigar e discutir sobre a } \\
\text { espiritualidade e as suas } \\
\text { intervenções nos cuidados } \\
\text { paliativos } \\
\text { desempenhada quando } \\
\text { enfermeiros. }\end{array}$ & $\begin{array}{l}\text { O estudo recomenda que inclua o } \\
\text { cuidado espiritual nos programas } \\
\text { educativos em enfermagem. O } \\
\text { cuidado físico é fundamental bem } \\
\text { como, o cuidado holístico do ser } \\
\text { humano em cuidados paliativos. }\end{array}$ \\
\hline 2018 & $\begin{array}{l}\text { ARRIEIRA } \\
\text { ICO, et al. }\end{array}$ & $\begin{array}{l}\text { Pelotas, } \\
\text { Brasil. }\end{array}$ & $\begin{array}{l}\text { Rev Esc } \\
\text { Enferm da } \\
\text { USP. }\end{array}$ & $\begin{array}{llr}\text { Entender sobre } & \text { a } \\
\text { espiritualidade } & \text { nos } \\
\text { cuidados paliativos no } \\
\text { âmbito da } & \text { equipe } \\
\text { interprofissional. } & \end{array}$ & $\begin{array}{l}\text { Traz sentido ao trabalho quando } \\
\text { envolve a espiritualidade na } \\
\text { assistência durante os cuidados } \\
\text { paliativos. Torna-se um contribuinte } \\
\text { na construção de vínculos, entre } \\
\text { profissionais, pacientes e } \\
\text { familiares. }\end{array}$ \\
\hline
\end{tabular}

Fonte: Monteiro TBM, et al., 2021. 
Seguida da releitura dos casos elegidos a partir do formulário de coleta de dados específicos para o estudo, construíram-se duas categorias: Desempenho da enfermagem em oncologia: cuidado espiritual e Espiritualidade na formação profissional do enfermeiro. A seguir, as categorias para organizar os resultados obtidos no processo de desenvolvimento da revisão integrativa.

\section{Desempenho da enfermagem em oncologia: cuidado espiritual}

A saúde espiritual do profissional de enfermagem precisa ser encarada como um relevante valor de sistema de crenças que pode vir afetar o seu desempenho profissional. Visto que a sua saúde espiritual reflete em melhores práticas e consequentemente, em bem-estar conjunto (CHIANG YC, et al., 2015). Dessa forma, o profissional ao utilizar do artifício espiritual, pretende alcançar o seu bem-estar pelo fato da espiritualidade estar interligada ao sentido, sentimento de satisfação e prazer no seu ambiente de trabalho (ARRIEIRA ICO, et al., 2018).

Nessa perspectiva, um estudo ao avaliar médicos e enfermeiros, constatou que a enfermagem é a classe mais propensa a fornecer os cuidados espirituais aos seus pacientes (RODIN D, et al., 2015). Essa informação pode ser justificada por outro estudo, que considera a enfermagem uma profissão que desenvolve o cuidado mais direto ao paciente e, assim apresenta como responsabilidade um olhar holístico para as diversas dimensões como biológica, social, psicológica e especialmente, espiritual. É nesta perspectiva que se compreende a espiritualidade essencial para uma assistência de enfermagem qualificada (EVANGELISTA CB, et al., 2016).

O mesmo estudo identifica a espiritualidade como fonte de conforto, força e fé. E que, a partir do momento que essa dimensão é cativada entre os pacientes em processo de morte, reflete em melhorias no seu quadro clínico e facilita a aceitação da sua condição de finitude (EVANGELISTA CB, et al., 2016).

Dessa maneira, vale ressaltar que a enfermagem envolve uma herança espiritual próspera ao assistir o paciente de modo a contemplar o todo, ou seja, as suas multidimensionalidades. A espiritualidade se associa à prática da enfermagem ao reconhecer o profissional como o integrador da assistência holística e ao associar a saúde e o bem-estar às dimensões humanas espiritual e física, especialmente diante de seu processo de morte. Nesta conjuntura, o cuidado espiritual deve incluir singularidades como, saber ouvir, fornecer suporte espiritual, demonstrar interesse ao diálogo entre profissional e paciente, favorecer e encorajar os seus propósitos de vida, além de possibilitar a transcendência dos sentimentos expressos no processo saúdedoença (HSIEH SI, et al., 2020).

Contudo, é imprescindível considerar também as dimensões dos profissionais. Há estudos que trazem evidências de que os profissionais que aceitam a morte de seus pacientes assistidos com maior facilidade são aqueles espiritualizados. Em vista disso, tornam-se capazes de reduzirem ou até mesmo, cessarem a dor, a angústia e o medo remanescentes diante do insucesso de salvar a vida de seu paciente sob os seus cuidados (SILVA BS, et al., 2016).

Algumas pesquisas fortalecem informações discutidas baseadas em diversos autores e concepções, que apoiam a ideia da espiritualidade ser uma dimensão referente às experiências dos seres humanos. Na qual se expressam pela busca interior e profunda do ser pessoal e pelo significado, baseados nos seus princípios, valores e crenças que possibilitarão restaurar o real sentido da vida e possibilitar a relação entre o Divino, 0 meio externo e consigo mesmo (SIQUEIRA HCH, et al., 2017).

Isso também foi evidenciado em uma pesquisa realizada no sul do Brasil, em que foi mediante a ação das práticas de orações e prestação de assistências de maneira integral, que se tornaram recursos terapêuticos essenciais para proporcionar conforto, uma sobrevida digna e humanizada diante à morte. Além disso, essas ações auxiliaram os profissionais e os pacientes a entenderem de fato o processo de morte, na busca de um sentido para o sofrimento advindo da enfermidade (ARRIEIRA ICO, et al., 2018).

\section{Espiritualidade na formação profissional do enfermeiro}

O cuidado espiritual como uma dimensão pertencente da assistência holística de enfermagem, inserido no ambiente dos cuidados aos pacientes em finitude de vida, pode beneficiar tanto o seu ambiente de trabalho 
quanto outros meios, além de favorecer a educação e formação profissional (SIQUEIRA HCH, et al., 2017; RONALDSON S, et al., 2017).

É importante ressaltar que os recursos tecnológicos estão mais aprimorados atualmente, porém, podem refletir em uma redução da sensibilidade sobre os sentimentos dos seres humanos, comprometendo a relação entre os profissionais da saúde e seus pacientes. Em consonância, se faz importante incluir nas disciplinas curriculares a espiritualidade na formação de profissionais na área da saúde, pois estes no campo de prática, já terão conhecimento e ambiência sobre os aspectos espirituais para o planejamento e condução de suas ações, o qual favorece tanto a formação profissional quanto pessoal (REGINATO V, et al., 2016).

Dessa forma, inserir uma disciplina que aborde a temática da espiritualidade durante o cuidado à saúde, pode direcionar futuros profissionais com mais sensibilidade e, capazes de compreender as necessidades de seus pacientes no momento do processo saúde-doença. Logo, considerar a valorização da espiritualidade como um recurso em prol da melhoria na qualidade do cuidado prestado, vai de encontro à desumanização do sistema de saúde atual (REGINATO V, et al., 2016).

Torna-se relevante essa discussão tendo em vista que ainda se concentra uma assistência à saúde considerada inadequada ao perfil de paciente abordado no estudo, no qual, o foco está, na maioria das vezes, na busca pela recuperação da saúde, utilização de recursos invasivos e de expressiva tecnologia. Menosprezando o sentimento de sofrimento expresso pelo paciente submetido à tais intervenções que não contemplam o tratar de sintomas subjetivos humanos ao evidenciar apenas a sua dor física (MACIEL AMSB, et al., 2018).

Para que a humanização seja cumprida na prática é fundamental a promoção de debates desde o processo de formação do enfermeiro, de modo a valorizar o trabalho em equipe e a inserção de todos os sujeitos envolvidos como protagonistas no âmbito do cuidado à saúde (REGINATO V, et al., 2016).

Então, no contexto da contemporaneidade, é imprescindível a criação de projetos que envolvam a espiritualidade, considerada um horizonte promissor, desde a graduação à pós-graduação no âmbito da saúde, em busca do desenvolvimento de uma assistência mais humanizada (FREITAS FDS, FERREIRA MA, 2016; OLIVEIRA RA 2017). Consequentemente, envolver conversas sobre a morte e o seu processo associado à valorização da espiritualidade, traz amparo e conforto aos envolvidos (LIMA R, et al., 2018).

Como limitações do estudo tem-se a restrição, a equipe de enfermagem, enquanto o cuidado espiritual pode ser uma importante estratégia terapêutica para todas as áreas da saúde.

\section{CONSIDERAÇÕES FINAIS}

A percepção da espiritualidade está relacionada com o processo de trabalho, uma vez que diante de momentos de sofrimento fisiopsiquicosocial e espiritual, em processo de morte, favorece a relação dos profissionais de enfermagem consigo mesmos e com o outro. Logo, tem-se a espiritualidade como uma estratégia facilitadora para a formação de vínculos saudáveis que ao ser fomentada no cuidado promoverá a humanização das relações interpessoais. Isto posto, a espiritualidade é uma temática contemporânea e reflete à melhores práticas assistenciais ao paciente em processo de morte, em especial o oncológico, porém estimula a assistência e o campo científico a intensificarem ações e pesquisas que incluam a dimensão espiritual para uma melhor compreensão deste fenômeno em outros cenários e situações.

\section{REFERÊNCIAS}

1. ARRIEIRA ICO, et al. Espiritualidade nos cuidados paliativos: experiência vivida de uma equipe interdisciplinar. Rev. Esc. Enferm. USP. 2018; 52.

2. BASTOS RA, et al. Angústias Psicológicas Vivenciadas por Enfermeiros no Trabalho com Pacientes em Processo de Morte: Estudo Clínico-Qualitativo. Trends Psychol, Ribeirão Preto. 2018; 26(2):795-805.

3. BUBLOZ BK, et al. Perceptions of Nursing Professionals with Regards to the Suffering and its Coping Strategies in Oncology. Rev Fund Care Online. 2019; 11(3):599-606. 
4. CATRÉ MNC, et al. Espiritualidade: Contributos para uma clarificação dos conceitos. Análise Psicológica. 2016; 34(1):31-46.

5. CHIANG YC, et al. The impact of nurses' spiritual health on their attitudes toward spiritual care, professional commitment, and caring. Nurs Outlook. 2015.

6. EVANGELISTA CB, et al. Spirituality in patient care under palliative care: A study with nurses. Esc. Anna Nery. 2016; 20(1):176-182.

7. FREITAS FDS, FERREIRA MA. Humanization knowledge of undergraduate nursing students. Rev. Bras. Enferm. 2016; 69(2):261-8.

8. HSIEH SI, et al. Factors associated with spiritual care competencies in Taiwan's clinical nurses: A descriptive correlational study. J Clin Nurs. 2020;29(9-10):1599-1613.

9. KUBLER-ROSS E. Sobre a morte e o morrer: o que os doentes terminais têm para ensinar a médicos, enfermeiras, religiosos e aos seus próprios parentes. Editora Martins Fontes. 2017.

10. LIMA R, et al. Death and the dying process: we still need to talk about it. Reme, Rev. Min. Enferm. $2017 ; 21: 1040$.

11. LIMA R, et al. Death education: sensibility for caregiving. Rev. Bras. Enferm. 2018; 71, suppl 4:1779-84.

12. MACIEL AMSB, et al. The condition of spirituality in oncological nursing care. Rev enferm UFPE. 2018; 12(11):30249.

13. MIQUELETTO M, et al. Espiritualidade de famílias com um ente querido em situação de final de vida. Rev Cuidarte. 2017; 8(2):1616-1627.

14. OLIVEIRA RA. Saúde e espiritualidade na formação profissional em saúde, um diálogo necessário. Rev. Fac. Ciênc. Méd. Sorocaba. 2017; 19(2):54-5.

15. QUIJADA PDS, et al. Qualidade de vida relacionada à saúde de pacientes com câncer de próstata em tratamento de radioterapia: revisão integrativa de literatura. Arq. Cienc. Saúde UNIPAR, Umuarama. 2018; 22(3):199-204.

16. REGINATO V, et al. Espiritualidade e saúde: uma experiência na graduação em medicina e enfermagem. Rev. Poli. 2016; 14(1):237-255.

17. RODIN D, et al. Whose role? Oncology practitioners' perceptions of their role in providing spiritual care to advanced cancer patients. Support Care Cancer. 2015.

18. RONALDSON S, et al. Palliative care nurses' spiritual caring interventions: A conceptual understanding. Int J Palliat Nurs. 2017; 23(3).

19. RUIVO BARA, et al. Assistência de enfermagem na segurança do paciente na UTI: uma revisão integrativa da literatura.2020. Revista Eletrônica Acervo Enfermagem, 5, e5221.

20. SIQUEIRA HCH, et al. Spirituality in the health-illness-care of the oncological user process: nurse's outlook. Rev enferm UFPE online, Recife. 2017; 11(8):2996-3004. 